\title{
Partejar de primíparas: reflexos na amamentação
}

\author{
Childbirth care for primiparas: reflexes in breastfeeding \\ Cuidado al parto de primiparas: reflejos en la lactancia materna
}

Idalina Reis de Castro'; Mônica Cecília Pimentel de Melo"; Ramon José Leal de Morais 'II; Ana Dulce Batista dos Santos'v

\begin{abstract}
RESUMO
Objetivo: analisar, a partir da experiência de primíparas, a relação entre a assistência recebida durante o parto normal e o pósparto imediato e seus reflexos na amamentação. Método: qualitativo, descritivo, aprovado pelo Comitê de Ética, realizado em um Centro de Parto Normal, do polo Petrolina-Juazeiro, em 2018. Participaram 17 primíparas, através de entrevista semiestruturada, sendo os dados submetidos a análise temática de conteúdo. Resultados: a amamentação ocorreu positivamente, pois esse lócus é apropriado para essa prática, favorecendo-a de maneira natural. $\mathrm{O}$ apoio e a compreensão são primordiais e devem superar uma assistência focada apenas em informações rápidas, técnicas e generalizadas. Conclusão: os anseios e particularidades de cada puérpera precisam ser respeitados e atendidos, valorizando o investimento em políticas de saúde que enfatizem o cuidado humanizado para cada mulher. Logo, a educação em saúde precisa estabelecer reflexão, discussão e aprendizado nesse processo que transcende o biológico.
\end{abstract}

Descritores: Assistência; parto; aleitamento materno; pós-parto.

\section{ABSTRACT}

Objective: to analyze the relationship between the care received during normal delivery and the immediate postpartum and its effects on breastfeeding, from the experience of primiparous women. Method: qualitative and descriptive study, approved by the Ethics Committee, held in a Normal Birth Center in Petrolina-Juazeiro Center, Brazil, in 2018. Seventeen primiparous women participated through semi-structured interviews, treated by thematic content analysis. Results: breastfeeding occurred positively, as this locus is appropriate for this practice, favoring it naturally. Supporting and understanding are primordial and should go beyond assistance focused only on quick, technical and generalized information. Conclusion: the wishes and particularities of each postpartum woman need to be respected and met, valuing the increment in health policies that emphasize humanized care for each woman. Therefore, health education needs to establish reflection, discussion and learning in this process that transcends the biological.

Descriptors: Assistance; parturition; breast feeding; postpartum.

\section{RESUMEN}

Objetivo: analizar la relación entre la atención recibida durante el parto normal y el posparto inmediato y sus efectos sobre la lactancia materna, a partir de la experiencia de las mujeres primíparas. Método: estudio cualitativo y descriptivo, aprobado por el Comité de Ética, realizado en un Centro de Parto Normal en el Centro Petrolina-Juazeiro, Brasil, en 2018. Diecisiete mujeres primíparas participaron a través de entrevistas semiestructuradas, tratadas por análisis de contenido temático. Resultados: la lactancia materna ocurrió positivamente, ya que este lugar es apropiado para esta práctica, favoreciéndolo naturalmente. El apoyo y la comprensión son primordiales y deben ir más allá de la asistencia centrada solo en información rápida, técnica y generalizada. Conclusión: los deseos y particularidades de cada mujer posparto deben ser respetados y cumplidos, valorando el incremento en las políticas de salud que enfatizan la atención humanizada para cada mujer. Por lo tanto, la educación sanitaria necesita establecer reflexión, discusión y aprendizaje en este proceso que trasciende lo biológico.

Descriptores: Asistencia; parto; lactancia materna; posparto.

\section{INTRODUÇÃO}

A assistência recomendada pela enfermagem obstétrica e neonatal é centrada na humanização como ferramenta do cuidado à parturiente. À vista disso, implementou-se a Portaria 985/GM de 5 de agosto de 1999, assegurando o acesso a assistência ao parto nos Serviços do Sistema Único de Saúde (SUS), além de ações que visam a diminuição da morbimortalidade materno-infantil, humanização e melhoria na qualidade da assistência. Assim sendo, foi estabelecida a criação dos Centros de Parto Normal (CPN) para a assistência à mulher no ciclo gravídico, com a oferta de serviços humanizados e de qualidade, no cuidado ao parto normal ${ }^{1,2}$.

\footnotetext{
'Graduada em Enfermagem. Universidade Federal do Vale do São Francisco, Petrolina-PE, Brasil. E-mail: idalinarcastro@hotmail.com "Enfermeira. Doutora. Docente da Universidade Federal do Vale do São Francisco, Petrolina-PE, Brasil, E-mail: monquinamelo@gmail.com I'Enfermeiro. Mestre. Docente da Universidade Federal do Vale do São Francisco, Petrolina-PE, Brasil. E-mail: ramonmorais_dm@hotmail.com.

IVEnfermeira. Mestre. Docente da Universidade Federal do Vale do São Francisco, Petrolina-PE, Brasil. E-mail: anadulcebs@yahoo.com.br
} 
Nessa mesma trajetória, a Portaria 1459/11 instaurou o Programa Rede Cegonha com o intuito de executar ações de assistência ao parto e nascimento, a fim de garantir acesso integral, acolhimento e resolutividade ${ }^{3}$. Desta forma, as Boas Práticas de Atenção ao Parto e Nascimento contida no documento da Organização Mundial da Saúde (OMS) de 1996 e fomentada na Rede Cegonha garante os direitos da gestante e orienta os profissionais. A ênfase da iniciativa está em assegurar a parturiente o direito ao acompanhante durante o trabalho de parto e o pós-parto, o incentivo à autonomia da mulher no processo de escolha do posicionamento para dar à luz, assim como, garantir o contato imediato e prolongado entre ambos, o aleitamento materno na primeira hora e o clampeamento tardio do cordão umbilical, entre outras 4 .

Na expectação que a mulher dentro desse processo de assistência prestada ao parto vivencia condições peculiares, mas que estão associadas de acordo com o mecanismo fisiológico e com o modelo da assistência prestada ao parto, questiona-se: Como se deu a condução do parto normal e do pós-parto imediato de primíparas e seus reflexos sobre a amamentação? O objetivo foi analisar, a partir da experiência de primíparas, a relação entre a assistência recebida durante o parto normal e o pós-parto imediato e seus reflexos na amamentação.

A relevância do estudo está na possibilidade de depreender a significância das vivências no processo de trabalho de parto, nascimento e aleitamento, em primíparas, a fim de garantir uma assistência de qualidade, mediante suas peculiaridades, assim como, disseminar a efetividade e a importância da aplicabilidade das boas práticas de parto e nascimento para os centros de parto normal. Isto posto, contribui para que profissionais da saúde avaliem as condições vivenciadas pelas parturientes, de forma singular, considerando seus limites e desejos, como também coopera para a sensibilização nos serviços, por meio da educação em saúde, salientando a importância da assistência de qualidade no parto, nascimento e amamentação.

\section{REVISÃO DE LITERATURA}

A OMS determina como ação primacial, após o parto, e já estabelecida pelas Boas Práticas de Atenção ao Parto e Nascimento, o estímulo à amamentação após o nascimento, assim que possível, com contato pele a pele imediato e ininterrupto. Tal ação é embasada na relação mãe-bebê nos primeiros minutos de vida, na qual, a criança estabelece o contato pele a pele, e é posicionada sobre a mãe em estado de alerta para realizar a sucção do leite materno ${ }^{5-7}$.

Dessa forma, a amamentação, quando estabelecida de forma precoce, proporciona inúmeros benefícios, tais como: forte teor nutricional, redução da morbimortalidade neonatal, desenvolvimento das defesas imunológicas, aperfeiçoamento sensorial e cognitivo, consolidação de vínculos, além do prolongamento da amamentação exclusiva, até o $6^{\circ}$ mês, complementado até os 24 meses ou mais ${ }^{8}$.

Convergindo com a assertiva anterior, a prática do aleitamento, logo que possível, ainda apresenta alguns entraves para as instituições, muito embora, se obtenha a comprovação da importância e das vantagens para mãe e bebê. Desse modo, para que a amamentação se estabeleça precocemente, torna-se necessário a atuação conjunta dos profissionais, fundamentada cientificamente, alicerçada por competência, empatia e destreza manual, principalmente, no que concernem as primíparas ${ }^{8,9}$.

Constantemente, surgem situações as quais as primíparas não têm a possibilidade de externar suas vontades, intenções e condições de bem-estar para o estabelecimento pleno do aleitamento materno. Assim, muito além do papel de nutriz, as mulheres no pós-parto imediato apresentam condições de exaustão, dor, e comportamentos inesperados que podem prejudicar o curso da amamentação e que devem ser avaliados com cautela? .

\section{METOdOLOGIA}

Estudo qualitativo e descritivo ${ }^{10}$, realizado em um CPN, na Região Integrada de Desenvolvimento (RIDE), polo Petrolina e Juazeiro. A instituição é uma unidade voltada para o parto de risco habitual, no atendimento às mulheres que demonstram um processo gestacional fisiológico e com ausência de intercorrências ${ }^{11}$.

Elencou-se como critérios de inclusão: puérperas, adolescentes ou adultas, primíparas, recém-nascido (RN) que obteve avaliação de escala de apgar acima de 8 no primeiro e no quinto minuto de vida e pós-parto normal de 12 horas. Aplicou-se a entrevista semiestruturada para um grupo de 17 primíparas, número determinado pelo alcance da saturação teórica ${ }^{12,13}$. Desta forma, obteve-se a inclusão de 17 primíparas.

O roteiro de entrevista conteve questões de caracterização dos participantes: idade, estado civil, escolaridade, duração do trabalho de parto e tempo de internação na unidade. As entrevistas foram guiadas pelas seguintes questões: Como foi para você a experiência do parto? O recém-nascido foi colocado em contato pele a pele com você assim que nasceu? Conte-me mais sobre isso. Você conseguiu amamentar seu bebê assim que ele nasceu? Conte-me mais sobre isso. Aqui na maternidade você recebeu orientações sobre amamentação? Nesse momento como está a amamentação? Por quê? 
A coleta ocorreu entre novembro a dezembro de 2018, após aprovação pelo Comitê de Ética da Universidade Federal do Vale do São Francisco, parecer no: 2.997.164. Foi realizada previamente uma seleção por meio de prontuários, na qual foram avaliadas as puérperas internadas que contemplavam os critérios de inclusão préestabelecidos. Posteriormente, após o consentimento das mesmas, as entrevistas foram realizadas na enfermaria.

Os depoimentos foram gravados em áudio, formato $\mathrm{mp} 3$, e transcritos integralmente. As participantes foram identificadas por códigos P1, P2, e assim, sucessivamente. Após transcrição, o material empírico foi submetido à análise temática de conteúdo, organizada em três fases: pré-análise, etapa de ordenação, na qual ocorre a seleção do material a ser analisado, subsequentemente à exploração do material em que se realizou a codificação e a enumeração, e, em seguida, o tratamento dos resultados adquiridos, mediante a inferência e a interpretação em que os dados encontrados tiveram significados e validação ${ }^{14}$.

\section{RESULTADOS E DISCUSSÃo}

Participaram 17 primíparas, entre 18 a 24 anos, majoritariamente, casadas, com ensino médio incompleto, com tempo de trabalho de parto entre 8 a 12 horas e tempo de internação entre 12 a 24 horas. A seguir, as 4 categorias temáticas elencadas.

\section{Experiência do parto e suas nuances na amamentação}

Esta categoria aborda a percepção das puérperas quanto à vivência do parto normal e amamentação. Os depoimentos caracterizaram uma assistência prestada com acolhimento, boas práticas do parto e nascimento, assim como estímulo à amamentação precoce, sendo avaliada pelas mulheres como importante para o momento. Dessa forma, surgiram relatos de práticas úteis, citadas na diretriz nacional de assistência ao parto normal que devem ser estimuladas.

Receberam-me superbem, maravilhoso, pari na cama, eu escolhi, tive um bom cuidado, eu gostei da conduta. (P5)

Foi tudo ótimo, [...] Deram toda assistência que podiam me dar, elas me falaram das posições para parir, fui bem atendida, eu escolhi a posição que queria parir, pari de cócoras. (P7)

Nesse tocante, o acolhimento é uma prática presente em todas as relações de cuidado, nos encontros reais entre prestadores de cuidados em saúde e nos atos de receber e escutar as pessoas. É uma prática distintiva no processo de cuidado. Assim, acolher é oferecer acolhida, ofertar escuta, aceitar as diferenças, sentir a necessidade do usuário, independente do profissional realizá-lo ou do ambiente onde o usuário se encontra. É a atitude de "estar com" e "estar perto de," é incluí-lo diante das suas necessidades ${ }^{14}$.

À vista disso, o acolhimento assume importância para um processo humanizado, que implica em uma recepção atenta e calorosa, com ênfase nas angústias, inseguranças e queixas apresentadas por essas mulheres e que deve ser assegurada. Dessa maneira, é imprescindível o acolhimento inicial das parturientes e seus acompanhantes nos centros de parto normal como um método de amenizar o desconforto proveniente do curso do parto ${ }^{15}$.

Destarte, as boas práticas de cuidado durante o parto e nascimento estão inteiramente interligadas ao processo de humanização, uma vez que são capazes de proporcionar o protagonismo por parte das mulheres, com ênfase em práticas menos intervencionistas e com eficácia confirmada na condução do parto e nascimento, assim como redução da morbimortalidade materna ${ }^{16}$.

Diversas práticas são recomendadas pela diretriz nacional de assistência ao parto normal, entre elas: o direito a acompanhante de livre escolha em todo o curso do parto, o apoio físico emocional na assistência, a oferta de líquidos, a escolha de movimentação e o posicionamento para parir, a oferta de métodos não farmacológicos para amenizar o desconforto, o contato pele a pele imediato entre mãe e bebê, a orientação e o incentivo à amamentação na primeira hora de vida. Tais práticas reduzem o tempo de trabalho de parto, bem como estimulam o vínculo entre mãe e bebê, assim como proporcionam um processo parturitivo natural e com condições favoráveis ao aleitamento materno precoce ${ }^{16,17}$.

Essa categoria enfatizou a relevância do processo de acolhimento atrelado diretamente à humanização como ferramenta indispensável para formação de vínculos, bem como uma assistência holística, de maneira que sejam asseguradas a essas mulheres boas práticas de cuidado durante o processo parturitivo e pós-parto, dando ênfase ao empoderamento dessas mulheres, apoio físico, emocional e garantia de contato pele a pele imediato entre o binômio.

Nessa mesma categoria, ainda em relação à percepção das mulheres sobre a experiência vivenciada, a sensação de dor esteve explicitamente evidenciada por relatos negativos, atribuídos ao parto normal como um processo doloroso.

Dolorosa, [...] senti muita dor [...]. (P2)

Horrivel, (risos) por causa do sofrimento [...], rapaz muita dor, é chato demais, você sente dores em lugares que você pensa que nem existe. (P4) 
Embora a sensação de dor faça parte da condução fisiológica do parto, é atrelada a diversos aspectos culturais e psicológicos, como o medo. Assim, sendo o parto uma experiência singular, e que envolve diversos fatores, não significa que essa vivência envolva apenas sensações positivas ou somente negativas ${ }^{18}$.

Observa-se que a dor e o sofrimento surgem como questões inevitáveis ao processo de parto normal, atrelado a uma vivência desconhecida e imprevisível ${ }^{19}$. Dessa maneira, a assistência deve ser prestada com o intuito de amenizar os fatores estressantes vivenciados pela parturiente em todo o curso do parto, de forma que sejam garantidos conforto e segurança. Para tal processo, são incentivadas pela diretriz da assistência ao parto e nascimento as práticas que são úteis e devem ser encorajadas, tais como, uso de métodos não farmacológicos, dentre esses, técnicas de massagem, movimentação, banho morno de chuveiro, musicoterapia, exercícios respiratórios, que devem ser ofertados e de livre escolha para cada mulher, com intuito de diminuir o desconforto e proporcionar singularidade ${ }^{18,19}$.

\section{Contato pele a pele e os reflexos na amamentação durante o pós-parto}

Tal categoria permitiu o agrupamento de depoimentos referentes à experiência do contato pele a pele precocemente após o parto e sua relação com a amamentação na primeira hora de vida. Foi mencionado nos relatos contato imediato entre mãe e bebê, a formação de vínculo, a facilidade para amamentar, além de sentimentos como emoção e amor.

Foi colocado em cima de mim, quase choro. Consegui amamentar, foi fácil. (P3)

Foi colocado na mesma hora, passou uma hora em cima de mim, foi bom ter ele pela primeira vez em meus braços, uma emoção enorme, um amor enorme, consegui amamentar. (P12)

O contato pele a pele imediato consiste em colocar a criança, em condições normais, ativa e reativa, sem uso de roupas, apenas de um campo aquecido, de modo direto sobre a mãe logo após o parto, com o intuito de proporcionar melhor adaptação ao ambiente externo, fortalecer vínculos, assim como estimular e oportunizar a amamentação no pós-parto imediato ${ }^{20}$.

Essa conduta possibilita ao recém-nascido, incentivos sensórios por meio do contato, do cheiro e dos barulhos, que contribui para o vínculo entre mãe e bebê, bem como maior probabilidade de êxito na amamentação. Com essa interação, o contato pele a pele desenvolve sentimentos de amor, proteção, bem estar, aconchego e comodidade para os dois. Sentir o cheiro da mãe e do leite materno, contribui para que o bebê descubra o seio materno, facilitando o início do aleitamento. Do mesmo modo, as batidas do coração da mãe e o som da voz materna acalentam e possibilitam estabilidade emocional ao recém-nascido ${ }^{21}$.

Destarte, foi possível observar o incentivo e a existência dessa prática para promover a humanização no processo parturitivo, além da garantia de condutas menos intervencionistas e baseadas em práticas benéficas ao acompanhamento do parto e nascimento, que favoreçam esse contato imediato e suporte na amamentação, evidenciada nos relatos por sucesso em amamentar no pós-parto imediato.

Houve ainda relatos de mães que vivenciaram o contato pele a pele, mas apresentaram dificuldades para realizar a amamentação de imediato.

Elas botaram em cima de mim, passou um tempinho bom, eu gostei do jeito que elas deixaram ele, [...] Quando ele nasceu, ele não estava querendo pegar o bico do peito, não sei se é porque não tem, [...], no mesmo dia mesmo, ele pegou. (P7)

Passou uma hora em cima de mim, eu botava ele no peito, mas não pegava, foi difícil, ele veio pegar hoje, [...]. Mas eu botava e ele recusava. (P10)

Tais fatos podem estar associados a inúmeros processos, uma vez que a condução do aleitamento materno está associada a condições biopsicossociais, como também referentes à atuação dos profissionais de saúde incluídos em todo o processo $^{22-24}$. Nessa perspectiva, ressalta-se a auto eficácia como influência direta no processo de promoção de saúde na amamentação, de tal forma que a confiança é um fator contribuinte para a escolha de amamentar. Isso define o nível de motivação, de maneira que a confiança relacionada aos saberes e habilidades corroboram para o sucesso na amamentação ${ }^{25}$.

Nesse sentido, os relatos demonstraram intrinsicamente a necessidade de um manejo mais efetivo por parte dos profissionais quando as adversidades podem surgir em meio à complexidade do momento que é o aleitamento materno precoce.

\section{Amamentação e a relação com as orientações recebidas}

Esta categoria surgiu dos relatos das puérperas no que diz respeito aos esclarecimentos e auxílio dos profissionais no processo de amamentação na maternidade. De acordo com as puérperas foram realizadas orientações no pós-parto imediato tais como: importância da amamentação, técnicas para pega correta, técnicas para evitar ingurgitamento, cuidados com a criança pós-mamada. 
Veio uma mulher, me instruiu como massagear, como fazer o biquinho de peixe, como tem que pegar o menino. (P2)

Elas vieram no quarto, e disseram que na hora da amamentação não é bom usar a mão em tesoura, que é melhor em forma de $C$, que na forma de tesoura prende o leite e a respiração do bebê. (P14)

Embora o processo de amamentar esteja associado de forma intrínseca à mulher, tido como um ato natural, ele é também o resultado de fatores comportamentais, de forma que possa ser trabalhado e assimilado, principalmente, em mães de primeira viagem. Diante disso, faz-se necessário estimular, orientar e oferecer suporte para que as puérperas iniciem de forma precoce o aleitamento materno e perdure de maneira exclusiva até 06 meses de vida ${ }^{26,27}$.

Nessa perspectiva, salienta-se a relevância das orientações e oferta de ajuda por partes dos profissionais de saúde, visto que mediante o nascimento do recém-nascido, a mãe dará início à prática de amamentação, caso essa seja sua vontade. Assim, esse momento é primordial para a realização de orientações a cerca da importância do aleitamento materno exclusivo, da pega correta, amamentação em livre demanda, desmitificação de costumes populares, dos cuidados com a criança após a mamada, intervenções de correção de acordo com as necessidades de cada puérpera ${ }^{28}$.

Ainda nessa mesma categoria, foi possível observar relatos que evidenciam orientações resumidas, que demandam pouco tempo dos profissionais, ou ainda, que não receberam nenhuma orientação sobre amamentação no CPN.

Na hora não, mas em casa minha sogra me ensinava direito. (P4)

Não vou mentir não, não recebi orientação não. (P7)

Vale salientar que o apoio de maneira acolhedora, em que o profissional mostre preocupação e interesse pela puérpera, é fundamental para incentivo e sucesso no processo de amamentar, dado que, o aleitamento está estreitamente relacionado com orientações, instruções, técnicas e, principalmente, pelo processo de escuta e conjuntura na qual a mãe está inserida ${ }^{29}$.

Nessa conjuntura, o suporte e a orientação às puérperas e à família necessitam de engajamento dos profissionais a fim de assegurar a prática efetiva de aleitamento, bem como, incentivo, salientando os benefícios e a relevância, visto que é um método natural, de baixo custo e de eficiência para mãe e bebê ${ }^{30}$.

Destarte, é relevante que os profissionais analisem o contexto da puérpera e que demonstrem comprometimento com as práticas que devem ser estimuladas para influenciar de forma positiva a lactação.

\section{Desfecho da amamentação no CPN}

No que se remete ao desfecho da amamentação no CPN, os relatos evidenciaram a percepção das puérperas sobre o sucesso e as dificuldades na amamentação no pós-parto imediato, de maneira que, a maioria relatou facilidade no processo, experiência positiva, pega correta e relação prazerosa no ato de amamentar.

Está bom, fácil, ela está pegando direitinho o peito, um sentimento muito bom. (P1)

Pra mim está fácil, eu não sinto dor quando ela suga, está prazeroso. (P6)

Em vista disso, mães que tiveram experiências positivas, possivelmente, terão maiores chances de estabelecer uma amamentação de forma imediata e contínua, quando correlacionada àquelas que vivenciaram situações negativas ${ }^{31}$.

Destaca-se que a amamentação é um processo a ser manejado, compreendido e apreendido pelas mães e que no decorrer, ela precisa ser influenciada, ter apoio e assistência direta. Mães inexperientes que não recebem suporte técnico e emocional encontram-se mais vulneráveis à descontinuidade da amamentação, salientando a necessidade de uma formação de rede de apoio para entender as demandas e as particularidades vivenciadas por cada mulher ${ }^{32}$.

Outro fator importante observado foi a pega correta, que reflete em uma sucção adequada e, consequentemente, em um maior estímulo à produção do leite e sensação de bem-estar e prazer, uma vez que, ocasiona a estimulação da ocitocina, além dos benefícios para nutrição e saciedade do recém-nascido ${ }^{32}$.

Isto posto, entende-se que o CPN é um ambiente adaptado para que a assistência ao parto e nascimento ocorra da forma mais natural possível, baseada na humanização e no bem-estar mãe e bebê, e que o puerpério imediato é o momento crucial para um desfecho positivo nesse processo, uma vez que é nele que ocorrem as maiores dificuldades. Por isso deve-se garantir que ocorra uma orientação de maneira completa e contínua, e que o profissional esteja alerta as especificidades de cada lactante, incentivando e promovendo o aleitamento materno precoce.

Em contrapartida, houve relatos de dificuldades para amamentar, sendo citados pelas mães como pega incorreta e carência de orientação pela equipe de saúde.

Está difícil amamentar, por eu não saber se ele está se alimentando ou não, ele puxa e não sai nada, sai esse leite fraquinho, [...]. (P1) 
Sabe-se que as primíparas podem demonstrar dificuldades significativas em relação à amamentação por não apresentarem experiências precedentes, logo, no puerpério imediato faz-se necessário maior suporte e orientação ${ }^{33}$. Todavia, observou-se que há falhas no processo assistencial do puerpério imediato, relativo ao aleitamento materno, uma vez que alguns profissionais realizam as orientações de maneira parcial. Dessa maneira, considerando que o ambiente possui características que favorecem a amamentação de forma plena, faz-se necessária equidade para um atendimento acolhedor e humanizado em todo o processo do ciclo lactacional.

\section{CONCLUSÃO}

Foi possível identificar que o processo de amamentar no CPN ocorreu de maneira satisfatória para a maioria das mães, sendo uma experiência positiva, prazerosa e de facilidade na prática. Evidenciou-se que o parto em CPN contribui para que essa prática aconteça de maneira natural e de forma imediata, uma vez que esse espaço proporciona proximidade entre mãe e bebê e fortalecimento do vínculo. Além disso, garante, por meio da assistência de qualidade dos profissionais, que os anseios e particularidades de cada puérpera sejam respeitados e sanados nesse momento crucial para a continuidade da amamentação. Constata-se que não são apenas orientações técnicas de forma rápida e única que as puérperas necessitam, mas de apoio e compreensão, estabelecendo proximidade e encurtando caminhos.

Torna-se relevante manter o investimento em políticas de saúde, enfatizando o cuidado humanizado, holístico e as necessidades de cada mulher. É necessário sensibilidade por parte dos profissionais de saúde, além do uso da educação em saúde para proporcionar reflexão, discussão e aprendizado nesse processo que transcende o biológico.

Em relação à limitação encontrada, cita-se a escassez de primíparas nesse período, que implicou na postergação de prazos, assim como, o reduzido número de participantes, em um único campo de pesquisa.

Mesmo assim, a pesquisa contribui para que os profissionais de saúde avaliem e compreendam as peculiaridades da mulher, considerando seus ensejos, como também coopera para a sensibilização dos demais profissionais e serviços por meio da educação em saúde, e salienta a relevância de uma assistência de qualidade no parto, nascimento e amamentação.

\section{REFERÊNCIAS}

1. Rocha FR, Melo MC, Medeiros GA, Pereira EP, Boeckmann LMM, Dutra LMA. Analysis of care to the mother-baby binomial in a center for normal delivery. Cogitare enferm. Rev. [internet]. 2017 [cited 2019 Jan 11]; 22(2):1-8. DOI:

http://dx.doi.org/10.5380/ce.v22i2.49228.

2. Silva TC, Bisognin P, Prates LA, Bortoli CFC, Oliveira LBR. Labor and birth care pratices an integrative review. Rev. enferm. Cent.Oeste Min. [Online]. 2017 [cited 2019 Mar 06]; 7(1):1-8. DOI: https://doi.org/10.19175/recom.v7i0.1294

3. Ministério da saúde (Br). Portaria no 1.459 de 24 de junho de 2011. Institui no âmbito do sistema único de saúde - SUS - a rede cegonha. Brasília (DF): Ministério da saúde; 2011 [cited 2019 Jan 11]; Available from: http://bvsms.saude.gov.br/bvs/saudelegis/gm/2011/prt1459_24_06_2011.html.

4. Ministério da saúde $(\mathrm{Br})$. Secretaria de atenção à saúde. Departamento de ações programáticas estratégicas, área técnica de saúde da criança e aleitamento materno. Área técnica de saúde da mulher, gravidez, parto e nascimento com saúde, qualidade de vida e bem-estar. Brasília (DF): Ministério da saúde; 2013 [cited 2019 Jan 11]; Available from: http://bvsms.saude.gov.br/bvs/publicacoes/gravidez_parto_nascimento_saude_qualidade.pdf.

5. Motta SAMV, Feitosa DS, Bezerra STF, Dodt RCM, Moreira DJ. Implementation of humanized care to natural childbirth. Rev. enferm. UFPE on line. [Online]. 2016 [cited 2019 Mar 05]; 10(2):593-9. Available from: https://periodicos.ufpe.br/revistas/revistaenfermagem/article/view/10994.

6. Carvalho MR. Nova revisão dos dez passos para o sucesso do aleitamento. In: OMS. Revisão da IHAC/BFHI mantém os 10 passos para o aleitamento materno. Tradução livre em processo de revisão/adaptação para o Brasil. 2018.

7. Melo RS, Costa ACPJ, Santos LH, Saldan PC, Santos Neto M, Santos FS. Exclusive breastfeeding practices among health professionals of a baby friendly accredited hospital. Cogitare enferm. Rev. [internet]. 2017 [cited 2019 Mar 05]; 22(4):1-14. DOI: https://dx.doi.org/10.5380/ce.v22i4.50523.

8. Vieira AC, Costa AR, Gomes PG. Good practices about breastfeeding: Application of the form of observation and assessment of suckling. Rev. Soc. Bras. Enferm. Ped. [internet] 2015 [cited 2019 Mar 05]; 15(1):13-20. Available from: https://sobep.org.br/revista/images/stories/pdf-revista/vol15-n1/vol_15_n_2-artigo-de-pesquisa-2.pdf.

9. Netto A, Spohr FA, Zilly A, França AFO, Rocha-Brischiliari SC, Silva RMM. Breastfeeding in the first hour of life at an institution with the baby-friendly hospital initiative. Ciênc. cuid. saúde. [Online]. 2016 [cited 2019 Jan 11]; 3(15):515-21. DOI: https://doi.org/10.4025/cienccuidsaude.v15i3.31508.

10. Kerr LRFS, Kendall C. A pesquisa qualitativa em saúde. Rev. Rene [internet]. 2013 [cited 2019 Mar 15]; 14(6):1061-3. Available from: http://periodicos.ufc.br/rene/article/view/3708.

11. Jamas MT, Hoga LAC, Rebert LM. Women's narratives on care received in a birthing center. Cadernos de Saúde Pública [Online]. 2013 [cited 2019 mar 15]; 29(12):2436-46. Available from: https://www.scielosp.org/scielo.php?pid=S0102311X2013001700009\&script=sci_arttext. 
12. Minayo MCS. Sampling and saturation in qualitative research: consensuses and controversies. [Online]. 2017 [cited 2018 Mar 06]; 5(7):1-12. Available from: https://edisciplinas.usp.br/pluginfile.php/4111455/mod_resource/content/1/Minayosaturacao.pdf.

13. Boni V, Quaresma SJ. Aprendendo a entrevistar: como fazer entrevistas em ciências sociais. Em Tese. [Online]. 2005 [cited 2018 Mar 06]; 2(1):68-80. Available from: https://periodicos.ufsc.br/index.php/emtese/article/download/18027/16976

14. Bardin, L. Análise de conteúdo. São Paulo: Edições 70; 2016.

15. Ministério da saúde $(\mathrm{Br})$. Secretaria de atenção à saúde. Departamento de atenção básica. Atenção ao pré-natal de baixo risco. Ministério da saúde. Secretaria de atenção à saúde. departamento de atenção básica. Brasília (DF): Ministério da saúde; 2012 [cited 2018 Mar 06]; Available from: http://bvsms.saude.gov.br/bvs/publicacoes/cadernos_atencao_basica_32_prenatal.pdf

16. Apolinário D, Rabelo M, Wolff LDG, Souza KSRR, Leal GCG. Practices in delivery and birth care from mothers' perspective. Rev. Rene. [Online]. 2016 [cited 2019 Mar 06]; 17(1):20-8. DOI: https://doi.org/10.15253/2175-6783.2016000100004.

17. Andrade LFB, Rodrigues QP, Silva RCV. Good Partices in obstetric care and its interface with humanization of assistance. Rev. enferm. UERJ. [Online]. 2017 [cited 2019 Feb 05]; 25:e26442:1-7. DOI: https://doi.org/10.12957/reuerj.2017.26442.

18. Ministério da saúde (Br). Secretaria de ciência, tecnologia e insumos estratégicos. Departamento de gestão e incorporação de tecnologias em saúde. Diretrizes nacionais de assistência ao parto normal: Ministério da saúde, secretaria de ciência, tecnologia e insumos estratégicos, departamento de gestão e incorporação de tecnologias em saúde. Brasília (DF): Ministério da saúde; 2017 [cited 2019 Feb 05]; Available from: http://bvsms.saude.gov.br/bvs/publicacoes/diretrizes_nacionais_assistencia_parto_normal.pdf

19. Feitosa RMM, Pereira DR, Souza TJCP, Freitas RJM, Cabral SAR, Souza LFF. Factors that influence the choice of birth type regarding the perception of puerperal women. Rev. pesqui. Cuid. fundam. [Online]. 2017 [cited 2019 Nov 03]; 9(3):717-26. DOI: https://dx.doi.org/10.9789/2175-5361.2017.v9i3.717-726 .

20. Siebra MA, Brito RC, Monteiro DMS, Monte NL. A dor do parto normal: significados atribuídos pelas puérperas usuárias do SUS. R. Interd. [Online]. 2015 [cited 2019 Feb 10]; 8(2):86-93. Available from: https://revistainterdisciplinar.uninovafapi.edu.br/index.php/revinter/article/download/639/pdf_224.

21. Almeida JM, Acosta LG, Pinhal MG. The knowledge of puerperae about non-pharmacological methods for pain relief during childbirth. REME rev. min enferm. [Online]. 2015 [cited 2019 Mar 06]; 19(3):711-17. DOI: https://dx.doi.org/10.5935/14152762.20150054.

22. Sampaio AS, Bousquat A, Barros C. Skin-to-skin contact at birth: a challenge for promoting breastfeeding in a "Baby Friendly" public maternity hospital in Northeast Brazil. Epidemiol. Serv. Saúde. [Online]. 2016 [cited 2019 Mar 06]; 25(2):281-90. Available from: http://www.scielo.br/scielo.php?pid=S2237-96222016000200281\&script=sci_arttext\&tlng=en.

23. Silva CM, Pereira SCL, Passos IR, Santos LC. Factors associated with skin to skin contact between mother/son and breastfeeding in the delivery room. Rev. Nutr. [internet]. 2016 [cited 2019 Mar 06]; 29(4):457-71. DOI: https://dx.doi.org/10.1590/167898652016000400002.

24. Mesquita AL, Souza VAB, Moraes Filho IM, Santos TN, Santos OP. Nursing roles in mothers' guidance about breastfeeding. Rev. Cient. Sena Aires. [Online]. 2016 [cited 2019 Mar 06]; 5(2):158-70. Available from: https://revistafacesa.senaaires.com.br/index.php/revisa/article/download/267/140.

25. Dominguez CC, Kerber NPC, Rockembach JV, Susin LRO, Pinheiro TM, Rodrigues EF. Difficulties in establishing breastfeeding: view of nurses working in primary care facilities. Rev. enferm. UERJ. [Online]. 2017 [cited 2019 Nov 03]; 25:e14448:1-6. DOI: https://doi.org/10.12957/reuerj.2017.14448.

26. Uchoa JL, Rodrigues AP, Joventino ES, Almeida PC, Oriá MOB, Ximenes LB. The self-efficacy in breastfeeding of women in the prenatal and postpartum: longitudinal study. Rev. Enferm. UFSM. [Online]. 2016 [cited 2019 Nov 03]; 6(1): 10-20. DOI: https://dx.doi.org/10.5902/2179769217687.

27. Cruz MR, Sebastião LT. Breastfeeding in premature infants: mothers'knowledge, feelings and experiences. Distúrbios Comun. [Online]. 2015 [cited 2019 Mar 06]; 27(1):76-84. Available from: https://revistas.pucsp.br/dic/article/download/19362/16328.

28. Barbieri MC, Bercini LO, Brondani KJM, Ferrari RAP, Grubisich MT, Tacla M, Sant'anna FL. Breastfeeding: guidance received in prenatal care, delivery and postpartum care. [Online]. 2015 [cited 2019 Feb 27]; 36(1):17-24. DOI: http://dx.doi.org/10.5433/1679-0367.2015v36n1Suplp17.

29. Alves FM, Oliveira TRH, Oliveira GKS, Santos GM. Knowledge of inhered puerpers in a joint accommodation about maternal breastfeeding. Revista SUSTINERE. [Online]. 2017 [cited 2019 Nov 03]; 5(1):24-37. DOI: https://dx.doi.org/10.12957/sustinere.2017.27321.

30. Amaral LJX, Sales SS, Carvalho DPSRP, Cruz GKP, Azevedo IC, Ferreira Júnior MA. Factors that influence the interruption of exclusive breastfeeding in nursing mothers. Rev. gaúch. enferm. [Online]. 2015 [cited 2019 Mar 06]; 36(spe):127-34. DOI: https://dx.doi.org/10.1590/1983-1447.2015.esp.56676.

31. Batista MR, Valeda, AA, Coelho DF, Cordova FP Health. Professionals' guidance on breastfeeding: the look of mothers. J. Nurs Health [internet]. 2017 [cited 2019 Feb 27]; 7(1):25-37. DOI: https://dx.doi.org/10.15210/jonah.v7i1.7718.

32. World health organization [internet]. geneva: baby-friendly hospital initiative; 2018. Ten steps to successful breastfeeding (revised 2018). [cited 2019 abr 15]; Available from: https://www.who.int/nutrition/bfhi/ten-steps/en/.

33. Hernandes TA, Fujinami AN, Raimundo EC, Cardoso CP, Higa EFR, Lazarini CA. Nursing mothers' social representation about breastfeeding. Revista Psicologia, Diversidade e Saúde. [internet]. 2017 [cited 2019 Feb 27]; 6(4):247-57. DOI: https://dx.doi.org/10.17267/2317-3394rpds.v6i4.1692. 